\title{
Accurately and Efficiently Studying the RF Structures Using a Conformal Finite-Difference Time-Domain Particle-in-Cell Method
}

\author{
M.C. Lin ${ }^{*}$, C. Nieter, P.H. Stoltz and D.N. Smithe \\ Tech-X Corporation, Boulder, Colorado, USA
}

\begin{abstract}
This work introduces a conformal finite difference time domain (CFDTD) particle-in-cell (PIC) method to accurately and efficiently study electromagnetic or radio frequency (RF) structures and their interactions with charged particles. For illustration, the dispersion relation of an A6 relativistic magnetron has been determined and a preliminary hot test including electrons has been done. The accuracy of the CFDTD method is measured by comparing with calculations based on the finite element method. The results show that an accuracy of $99.4 \%$ can be achieved by using only 10,000 mesh points with the Dey-Mittra algorithm as implemented in the CFDTD method. By comparison, a mesh number of 250,000 is needed to preserve $99 \%$ accuracy using a staircased FDTD method. This suggests one can more efficiently and accurately study the hot tests of microwave tubes or the interactions of charged particles and RF structures using the CFDTD PIC method than a conventional FDTD one.
\end{abstract}

\section{INTRODUCTION}

The magnetron is one of the major microwave tubes in use today to generate microwaves. Its invention and development can be dated to World War II [1]. There are mainly two categories of magnetrons. One is conventional magnetrons in which voltages of a few hundred to tens of kilovolts are applied between the anode and a heated, thermionic cathode. Power levels above hundreds of kilowatts can be achieved with conversion efficiencies as high as $80 \%$. The other is relativistic magnetrons in which pulsed voltages of several hundred $\mathrm{kV}$ to $\mathrm{MV}$ are used to launch relativistic electrons from cold cathodes to generate microwaves at GW power levels. However, reduced efficiencies are always observed in the experiments of the relativistic magnetrons [2]. Although magnetrons are widely used as microwave sources, a fundamental understanding of the underlying interaction physics is still being developed, particularly in the nonlinear regime. Computer simulation studies provide a particularly valuable approach to the analysis of the interaction physics and nonlinear electrodynamics in magnetrons [3]. In recent years, the conventional finite difference time domain (FDTD) particle-in-cell (PIC) method employing staircased mesh cells has been widely used to study magnetrons as well as other microwave tubes [4, 5], and it is called a simulation study of hot tests when particles are included. However, the original Yee scheme does not produce second order accurate results for models with curved edges or surfaces due to the staircasing approximation [6].

In this work, the conformal finite difference time domain (CFDTD) PIC method [7], [8] as implemented in VORPAL [9] has been employed to determine the dispersion relation of an A6 relativistic magnetron and study a hot test. The A6 relativistic magnetron in [3] is used for demonstration. The

*Address correspondence to this author at the Tech-X Corporation, Boulder, Colorado, USA; E-mail: mclin@txcorp.com schematic of this model and the corresponding geometric parameters are shown in Fig. (1), where the anode is located at $b$ and the cathode is located at $a$. We have constructed a $3 \mathrm{D}$ model of this A6 magnetron in VORPAL, as shown in Fig. (2). The 3D model is for illustration and all calculations are $2 \mathrm{D}$. As there is no exact solution for this case, to measure the accuracy of the CFDTD method, we employed results from SUPERFISH calculations as a reference. We also compared the results from the conventional FDTD method [6]. It is demonstrated that the CFDTD method generates second order accurate results, compared with first order obtained with the staircased FDTD method.

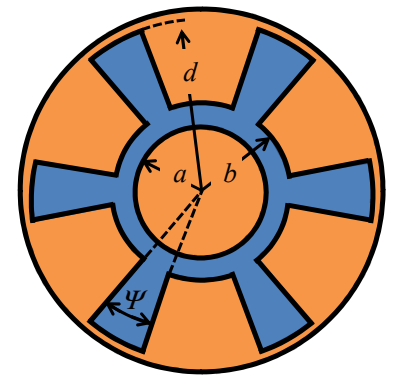

Fig. (1). Schematic of the A6 relativistic magnetron and the corresponding geometric parameters in [3], where $a=0.01448(\mathrm{~m})$, $b=0.03279(\mathrm{~m}), d=0.07279(\mathrm{~m})$, and $\Psi=20$ degrees.

\section{DETERMINATION OF DISPERSION RELATION FOR A6 MAGNETRON AND A HOT TEST STUDY}

To study microwave tubes numerically, one can do both a cold test and a hot test of the models. In the cold test, the dispersion relation of the interacting structures or slow wave structures is determined. This can be done in the frequency or time domain without putting in charged particles or electrons. The hot test is typically performed after acquiring the correct dispersion from the cold test of the model.

\subsection{Finite Element Based SUPERFISH Calculations}

Although the dispersion of the A6 magnetron can be obtained approximately using an admittance-matching 
method analytically developed during World War II [1], there is no exact solution for the case. The SUPERFISH code based on the finite element method (FEM) can be employed for the cold test to determine the resonances of the magnetron in the frequency domain [10]. Therefore, to measure the accuracy of the CFDTD method, we employed the results from SUPERFISH calculations as a reference. We have carefully tested the convergence of SUPERFISH calculations. A mesh number of 410,481 is large enough for a $2 \mathrm{D}$ problem to determine the dispersion of the A6 magnetron with a very high accuracy; however, one must perform separate calculations for each mode. In addition, SUPERFISH is a pure electromagnetic code and no particles are included in the calculations.

\subsection{Conventional FDTD Method}

To analyze a radio frequency (RF) structure with arbitrary geometries using a conventional FDTD method, staircased mesh cells with the Yee algorithm are employed to model the curved surfaces. The time evolution of Maxwell's equations is described via the conventional FDTD update equations. However, the procedure not only introduces errors due to inaccurate modeling of the geometry, but can possibly introduce spurious solutions [6, $11,12]$. Several approaches have been proposed to overcome these difficulties. One of them is the CFDTD method.

\subsection{CFDTD Simulation with Dey-Mittra Algorithm}

The CFDTD method is based on the Dey-Mittra algorithm [7, 8]. Locally distorted cells with edges tangential to the metallic surface are used. The fields in these distorted cells are computed using a modification of the conventional FDTD update equations. For each cell partially within the region of interest, the magnetic field is assumed to be located at the center of that undistorted Cartesian cell and constant over the area of the distorted cell. The electric fields are assumed to have a constant value along the edge of a cell that resides within the cavity and are zero along the metallic surface. With these assumptions, one can use the regular FDTD equation for updating the magnetic field with the use of the electric field values along the distorted contour appropriately weighted with the lengths of the contours. Once the magnetic fields are computed, the electric fields are updated in the conventional manner with the use of the adjacent magnetic field values. For the CFDTD method implemented in VORPAL, the DMVEFRA parameter sets the fraction of Courant time for which the simulation will be stable and the size of distorted cells to be included in the simulations [9]. The maximum stable time step when using these boundary conditions can be arbitrarily small, due to the presence of small fractional cells inside the vacuum region. Use of the Gershgorin Circle theorem allows the determination of a rigorous criterion for exclusion of small cells in order to have numerical stability for particular values of the ratio DMV̈FRAC $=\Delta \mathrm{t} / \Delta \mathrm{t}_{\text {Courant }}$ of the Courant time step [13].

\subsection{CFDTD Particle-in-cell Method}

The conformal finite-difference time-domain particle-incell method employs both the Dey-Mittra algorithm mentioned above and the particle-in-cell algorithm.

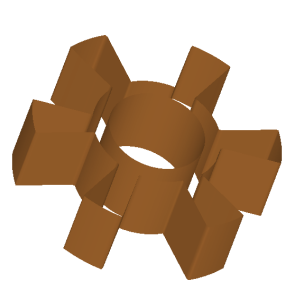

(a)

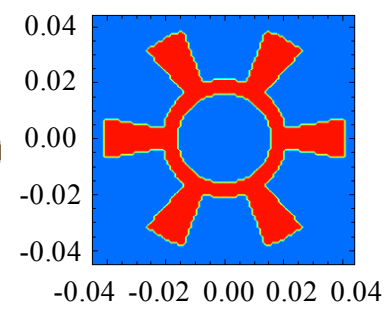

(b)
Fig. (2). Model of the A6 relativistic magnetron constructed in VORPAL, (a) 3D view and (b) 2D cross section view.

Beginning from a specified initial state, a physical process involving interactions between charged particles and electromagnetic fields can be simulated as it evolves in time. The full set of time-dependent Maxwell's equations is solved to obtain electromagnetic fields using the CFDTD method. In the PIC scheme [4], individual particles in a Lagrangian frame are tracked in continuous phase space, whereas moments of the distribution such as densities and currents are computed simultaneously on Eulerian mesh points. However, both fields and particles are defined at discrete times and they are advanced sequentially in time, starting from initial conditions. The complete Lorentz force equation is solved to obtain relativistic particle trajectories, using fields interpolated from the discrete grid to the continuous particle locations. Then, the current and charge densities are accumulated from the continuous particle locations to the discrete mesh locations for Maxwell's equations. The fields are then advanced one timestep. This self-consistent approach is commonly referred to as the EM PIC method, and is suitable for dealing with the interaction between charged particles and electromagnetic fields. The main attractive feature of the CFDTD PIC method is that the electromagnetic fields are more accurately described through the Dey-Mittra algorithm compared to the conventional Yee's algorithm. However, the bookkeeping involved is relatively simple as in the conventional FDTD method compared to other explicit nonorthogonal FDTD schemes [7, 8]. However, special treatment of particles at the cut-cell boundaries has to be made and emission and absorption algorithms preserving charge conservation have been developed $[14,15]$.

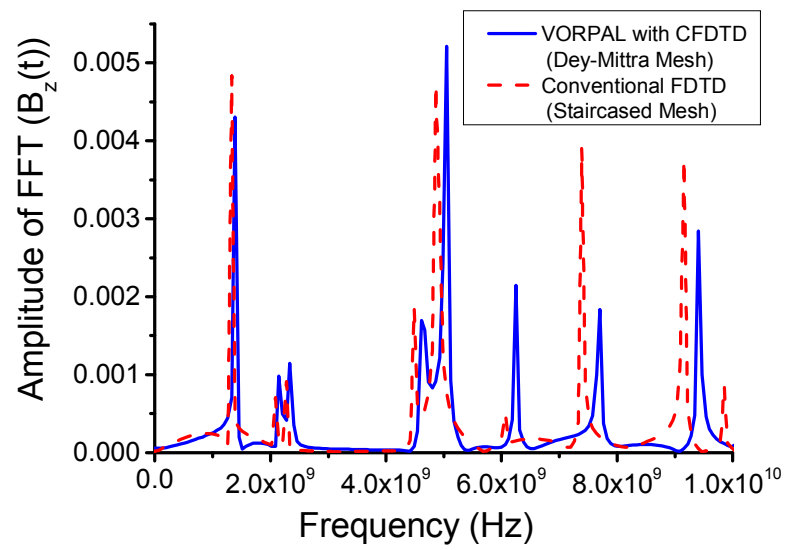

Fig. (3). Frequency shift of the A6 relativistic magnetron between the conventional FDTD method and the CFDTD method. Here $100^{2}$ mesh points are used for both methods. 


\section{SIMULATION RESULTS AND DISCUSSION}

The seven resonances in the first two bands of the A6 magnetron cavity determined by the SUPERFISH calculations are listed in Table 1. The accuracy of the CFDTD method reported here is measured by comparing with SUPERFISH calculations. In the time domain simulations of the cold test, we excite an impulse of current loop in the left-hand side cavity and measure the fields at the right-hand side cavity of the model shown in Fig. (2). The time domain data of the $z$ component of magnetic field $B_{z}(t)$ are measured and the corresponding frequency spectrum can be obtained by doing a fast Fourier transform (FFT), as shown in Fig. (3). All the resonances can be determined in one simulation run.

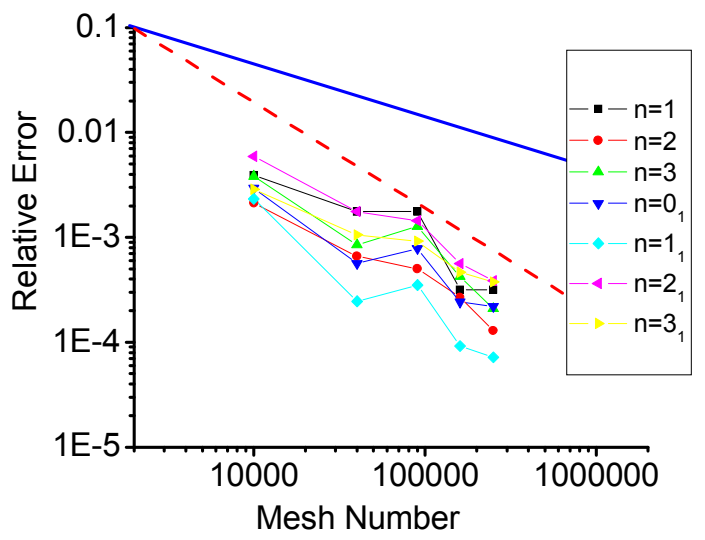

Fig. (4). Convergence test of the CFDTD method with DMVFRAC $=0.5$ on a simulation of an A6 magnetron cavity. Total simulation time is $1,000 \mathrm{~ns}$ for each case. The solid line and dashed line represent first and second order accuracy, respectively.

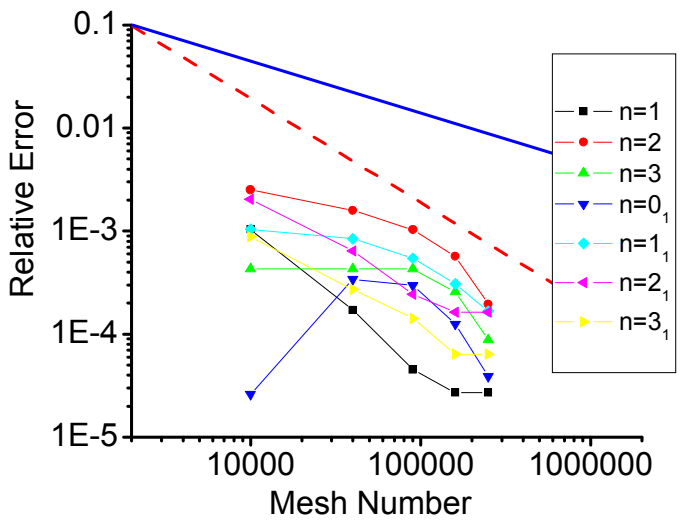

Fig. (5). Convergence test of the CFDTD method with DMVFRAC $=0.25$ on a simulation of an A6 magnetron cavity. Total simulation time is $1,000 \mathrm{~ns}$ for each case. The solid line and dashed line represent first and second order accuracy, respectively.

To benchmark the CFDTD method, we used different mesh numbers of $100^{2}, 200^{2}, 300^{2}, 400^{2}$, and $500^{2}$ with two DMVFRAC values equal to 0.5 and 0.25 , respectively, and all the calculations with uniform mesh cells were done on an AMD Opteron PC cluster with 32 nodes. The total simulation time for each case is $1,000 \mathrm{~ns}$ and the corresponding resolution of frequency spectra from FFT is 1 $\mathrm{MHz}$. The results show that an accuracy of $>99 \%$ can be achieved by using only 10,000 mesh cells with Dey-Mittra algorithm, DMVFRAC $=0.5$, as shown in Fig. (4). The price is the time step used has to be reduced to half of that satisfying the Courant criterion. By further reducing the DMVFRAC values in VORPAL, the accuracy can be further improved but the time step has to be again decreased, e.g., by a factor of 2 for using DMVFRAC $=0.25$ compared with that employing DMVFRAC $=0.5$, as shown in Fig. (5). The dispersion relations predicted by VORPAL simulations and SUPERFISH calculations are in good agreement. By comparison, although the time step reduction is avoided, a mesh number of 250,000 need be used to preserve an accuracy of $99 \%$ using the conventional FDTD method, as shown in Fig. (6). One can also see from Fig. (3), a frequency shift of conventional FDTD results from CFDTD ones is obvious if only 10,000 mesh points are used. The CFDTD method approximately exhibits a second order accuracy (dashed line), as shown in Figs. $(4,5)$, while the conventional FDTD is just a first order accuracy (solid line), as shown in Fig. (6). The efficiency of the two methods can be seen by comparing the simulation parameters, time step, number of time step, and wall clock time of the three cases listed in Table 2 . One may note that the trend of the convergent curves of the CFDTD method shown in Figs. (4, 5) is not always going down as increasing mesh number. This is possibly caused by the exclusion of smaller cut-cells for different resolutions. In spite of this, all errors for the cases with Dey-Mittra algorithm are at least an order of magnitude smaller when compared to those of the staircased cases shown in Fig. (6).

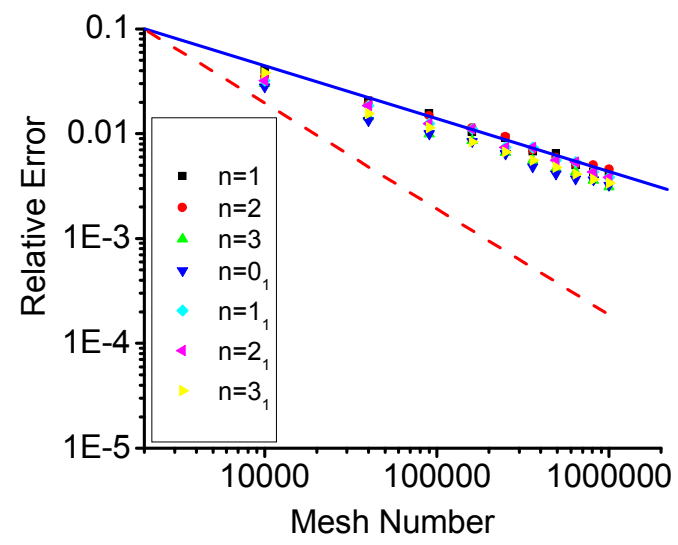

Fig. (6). Convergence test of the conventional FDTD method on a simulation of an A6 magnetron cavity. Total simulation time is 1,000 ns for each case. The solid line and dashed line represent first and second order accuracy, respectively.

To demonstrate the accuracy of VORPAL, we further compare the simulation results of the two cases corresponding to 10,000 and 40,000 mesh cells with DMVFRAC $=0.5$. One can see from Table 1 , by using the CFDTD method with only 10,000 mesh cells and DMVंFRAC value of 0.5 , we can determine the dispersion of the A6 magnetron within an accuracy of $99.4 \%$. By increasing the mesh cells to 40,000 , we can push the accuracy up to $99.8 \%$.

A preliminary hot test by using the CFDTD PIC method has been done. A high voltage of $325 \mathrm{kV}$ is applied between the anode and the cathode and a static magnetic field of $7 \mathrm{kG}$ is applied along the $z$ direction. In the hot test, the electrons are emitted from the cathode surface with a current density 
Table 1. Accuracy of VORPAL with Dey-Mittra CFDTD by Comparing with SUPERFISH Calculations. The Total Simulation Time is $1,000 \mathrm{~ns}$

\begin{tabular}{|c|c|c|c|c|c|}
\hline A6 Relativistic Magnetron Mode Number n (Ref. [3]) & $\begin{array}{c}\text { f(GHz) } \\
\text { SUPERFISH } \\
\# 410,481\end{array}$ & $\begin{array}{c}\text { f (GHz) VORPAL } \\
\text { (CFDTD) \# 10,000 } \\
\text { DM_FRAC }=0.5\end{array}$ & $\begin{array}{c}\text { Relative } \\
\text { Error }\end{array}$ & $\begin{array}{c}\text { f (GHz) VORPAL } \\
\text { (CFDTD) \# 40,000 } \\
\text { DM_FRAC }=0.5\end{array}$ & $\begin{array}{c}\text { Relative } \\
\text { Error }\end{array}$ \\
\hline 1 & 1.38443754 & 1.379 & 0.00393 & 1.382 & 0.00176 \\
\hline 2 & 2.15457799 & 2.150 & 0.00212 & 2.156 & 0.00066 \\
\hline 3 & 2.35399345 & 2.345 & 0.00382 & 2.352 & 0.00085 \\
\hline $0_{1}$ & 4.63362062 & 4.620 & 0.00294 & 4.631 & 0.00057 \\
\hline $1_{1}$ & 5.02876229 & 5.017 & 0.00234 & 5.030 & 0.00025 \\
\hline $22_{1}$ & 6.25401881 & 6.217 & 0.00592 & 6.243 & 0.00176 \\
\hline $3_{1}$ & 7.69109058 & 7.669 & 0.00287 & 7.683 & 0.00105 \\
\hline
\end{tabular}

Table 2. Comparison of the Simulation Parameters, Time Step, Number of Time Step, and Wall Clock Time for the Three Cases. The Total Simulation Time is $1,000 \mathrm{~ns}$

\begin{tabular}{|c|c|c|c|c|c|}
\hline Simulation \# & Mesh \# & DM_FRAC & Time sep $\Delta$ t & \# of Time Step & Wall Clock Time \\
\hline \hline Case 1 & $100^{2}$ & 0.50 & $8.14019 \mathrm{E}-13$ & $1,228,472$ & $31 \mathrm{~h} 21 \mathrm{~m} 33 \mathrm{~s}$ \\
\hline Case 2 & $100^{2}$ & 0.25 & $4.07001 \mathrm{E}-13$ & $2,456,944$ & $63 \mathrm{~h} 13 \mathrm{~m} 42 \mathrm{~s}$ \\
\hline Case 3 & $500^{2}$ & 1.00 & $3.25607 \mathrm{E}-13$ & $3,071,180$ & $86 \mathrm{~h} 26 \mathrm{~m} 27 \mathrm{~s}$ \\
\hline
\end{tabular}

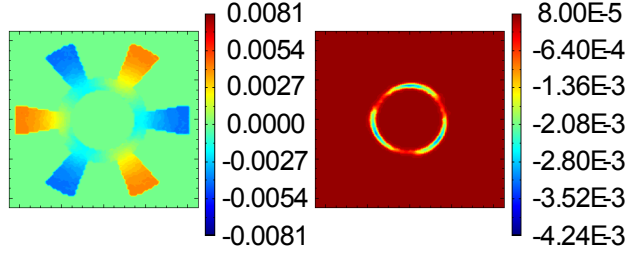

(a)

(b)
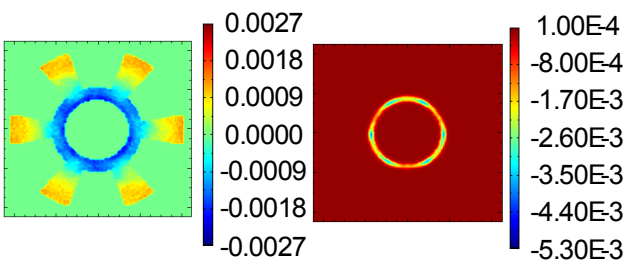

(c)

(d)

Fig. (7). Magnetic field patterns Bz, (a) and (c), for the $\pi(n=3)$ and $2 \pi\left(n=0_{1}\right)$ modes of the A6 magnetron, respectively, and the corresponding distributions of the electron space charge densities, (b) and (d).

of $1,000 \mathrm{~A} / \mathrm{m}^{2}$ and the impulse used in the cold test mentioned above has been removed. It is found that both $\pi(n=3)$ and $2 \pi\left(n=0_{1}\right)$ modes can be excited in the A6 magnetron, following the prediction of Buneman-Hartree theory [1]. The ac magnetic field patterns $B_{z}$ and the corresponding distributions of the electron space charge densities for the two modes of the A6 magnetron are shown in Fig. (7). The resonant frequencies obtained from the hot test with 10,000 mesh cells by doing FFT of the measured $B_{z}$ for the $\pi$ and $2 \pi$ modes are 2.340 and $4.628 \mathrm{GHz}$, respectively, and these results are very close to those from the cold test as the beam loading effect in this case is small. This forms a basis for us to study the interaction mechanisms of relativistic magnetrons with a small-signal analysis to investigate the low efficiency issue mentioned above. One can also study the large-signal nonlinear behaviors by simply increasing the current density to be thermionic or field emission limited or space-charge limited.

\section{CONCLUSION}

In this work, we have demonstrated that one can accurately and efficiently study RF structures using the CFDTD PIC method. The accuracy of the CFDTD method with the Dey-Mittra algorithm has been demonstrated by comparing with SUPERFISH calculations in which more than 400,000 mesh cells in 2D were employed. By using only 10,000 mesh cells in VORPAL, one can get results with an accuracy of more than $99.4 \%$. In addition, all the resonances in the electromagnetic structures can be determined in just one simulation run to form a correct dispersion relation of a slow wave structure of microwave tubes. One can expect that the simulation time of hot tests of microwave tubes or the interactions of charged particles and RF structures would be dramatically reduced by using a CFDTD PIC method without losing accuracy.

\section{ACKNOWLEDGEMENTS}

This work was supported in part by the U.S. Department of Defense under Grant No. FA9451-07-C-0025.

\section{REFERENCES}

[1] Collins GB. Microwave magnetrons. New York: McGraw-Hill 1948.

[2] Palevsky A, Bekefi G. Microwave emission from pulsed, relativistic e-beam diodes. II. The multiresonator magnetron. Phys Fluids 1979; 22: 986-96. 
[3] Davidson RC, Chan HW, Chen C, Lund S. Equilibrium and stability properties of intense non-neutral electron flow. Rev Mod Phys 1991; 63: 341-74.

[4] Verboncoeur JP, Langdon AB, Gladd NT. An object-oriented electromagnetic PIC code. Comput Phys Commun 1995; 87: 199211.

[5] Goplen B, Ludeking L, Smithe D, Warren G. MAGIC User's Manual, MRC/WDC-R-409, Mission Research Corp, Newington, VA 1997.

[6] Yee K. Numerical solution of initial boundary value problems involving Maxwell's equations in isotropic media. IEEE Trans Antennas Propag 1966; 14: 302-7.

[7] Dey S, Mittra R, Chebolu S. A technique for implementing the FDTD algorithm on a nonorthogonal grid. Microw Opt Technol Lett 1997; 14: 213-5.

[8] Dey S, Mittra R. A locally conformal finite-difference time-domain (FDTD) algorithm for modeling three-dimensional perfectly conducting objects. IEEE Microw Guided Wave Lett 1997; 7: 2735 .

[9] Nieter C, Cary JR. VORPAL: a versatile plasma simulation code. J Comput Phys 2004; 196: 448-73.
[10] Billen JH, Young LM. Poisson SUPERFISH, LANL Report No. LA-UR-96-1834, Los Alamos, NM 2003 (unpublished).

[11] Umashankar KR, Taflove A, Beker B. Calculation and experimental validation of induced currents on coupled wires in an arbitrary shaped cavity. IEEE Trans Antennas Propag 1987; AP-35: 1248-57.

[12] Jurgens TG, Taflove A, Umashankar K, Moore TG. Finitedifference time-domain modeling of curved surfaces. IEEE Trans Antennas Propag 1992; 40: 357-66.

[13] Nieter C, Cary JR, Werner GR, Smithe DN, Stoltz PH. Application of Dey-Mittra conformal boundary algorithm to 3D electromagnetic modeling. J Comput Phys 2009; 228: 7902-16.

[14] Mahalingam S, Nieter C, Loverich J, Smithe D, Stoltz P. Space charge limited currents calculations in coaxial cylindrical diodes using particle-in-cell simulations. Open Plasma Phys J 2009; 2: 639.

[15] Loverich J, Nieter C, Smithe D, Mahalingam S, Stoltz P. Charge conserving emission from conformal boundaries in electromagnetic PIC simulations. Comput Phys Commun 2009; in press.

This is an open access article licensed under the terms of the Creative Commons Attribution Non-Commercial License (http://creativecommons.org/licenses/by$\mathrm{nc} / 3.0 /$ ) which permits unrestricted, non-commercial use, distribution and reproduction in any medium, provided the work is properly cited. 